\title{
Knowledge Levels Regarding Crimean-Congo Hemorrhagic Fever Among Emergency Healthcare Workers in an Endemic Region
}

\author{
Sadiye Yolcu ${ }^{\mathrm{a}, \mathrm{d}}$, Cigdem Kader ${ }^{\mathrm{b}}$, Afsin Emre Kayipmaz ${ }^{\mathrm{c}}$, Sedat Ozbay ${ }^{\mathrm{c}}$, Ayse Erbay ${ }^{\mathrm{b}}$
}

\begin{abstract}
Background: In this study, we aimed to determine knowledge levels regarding Crimean-Congo hemorrhagic fever (CCHF) among emergency healthcare workers (HCWs) in an endemic region.
\end{abstract}

Methods: A questionnaire form consisting of questions about CCHF was applied to the participants.

Results: The mean age was $29.6 \pm 6.5$ years (range 19 - 45). Fiftyfour $(49.5 \%)$ participants were physicians, 39 (35.8\%) were nurses and $16(14.7 \%)$ were paramedics. All of the participants were aware of CCHF, and 48 (44\%) of them had previously followed CCHF patients. Rates of the use of protective equipment (masks and gloves) during interventions for patients who were admitted to the emergency service with active hemorrhage were $100 \%$ among paramedics, $76.9 \%$ among nurses and $61.1 \%$ among physicians $(\mathrm{P}=0.003)$. Among 86 (78.9\%) HCWs who believed that their knowledge regarding $\mathrm{CCHF}$ was adequate, $62(56.9 \%)$ declared that they would prefer not to care for patients with $\mathrm{CCHF}(\mathrm{P}=0.608)$.

Conclusions: The use of techniques to prevent transmission of this disease, including gloves, face masks, face visors and box coats, should be explained to emergency room HCWs, and encouragement should be provided for using these techniques.

Keywords: Crimean-Congo hemorrhagic fever; Emergency; Healthcare worker

Manuscript accepted for publication March 11, 2014

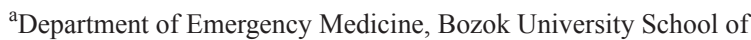
Medicine, Yozgat, Turkey

${ }^{b}$ Department of Infectious Diseases, Bozok University School of Medicine, Yozgat, Turkey

${ }^{c}$ Department of Emergency Medicine, Sivas Numune Hospital, Sivas, Turkey

${ }^{\mathrm{d}}$ Corresponding author: Sadiye Yolcu, Department of Emergency Medicine, Bozok University School of Medicine, Yozgat, Turkey. Email: sadiyeyolcu@yahoo.com

doi: http://dx.doi.org/10.14740/jocmr1801w

\section{Introduction}

Crimean-Congo hemorrhagic fever (CCHF) was first described in 1944 [1]. The CCHF virus is transmitted to humans via the bites of infected ticks or by direct contact with the secretions or blood of infected animals or humans. CCHF is a potentially fatal infection. It is endemic in over 30 countries around the Black Sea and in the Middle East and Africa [2]. CCHF infections were first reported in Turkey in 2003 among individuals who became sick in $2002[3,4]$.

In endemic regions, individuals who have occupational contact with livestock and wild animals, including shepherds, farmers and veterinarians, are at high risk for CCHF $[5,6]$.

Similarly, healthcare workers (HCWs) caring for CCHF patients are the second major group at risk for infection [7]. In Turkey, the neighboring cities of Sivas and Yozgat are endemic sites for CCHF. The climate of these cities is suitable for the survival of ticks, and the first cases of CCHF virus infection in Turkey were reported in this region [3].

HCWs are at risk for blood- and secretion-borne pathogens. Beltrami et al reported that at least 20 pathogens can be transmitted by needle sticks or sharps injuries. These pathogens can be transmitted to HCWs via blood and secretions [8]. Emergency HCWs are also at risk for these infectious diseases [9]. Outbreaks of CCHF among HCWs have been frequently reported and have a high mortality. The highest risk of transmission is from percutaneous exposure [10-14].

In this study, we aimed to determine knowledge levels regarding CCHF among emergency service (ES) HCWs in the cities of Sivas and Yozgat, where CCHF is endemic.

\section{Materials and Methods}

After approval by the local ethics committee, the study was conducted at the Sivas Numune Hospital Emergency Service, the Sivas Government Hospital Emergency Service and the Yozgat Government Hospital Emergency Service. A total of 109 HCWs (54 doctors, 39 nurses and 16 paramedics) received a questionnaire. Data regarding the age, gender 
Table 1. Questions Asked to Participants

Q1 Have you ever heard of CCHF?

Q2 Do you think that your CCHF knowledge level is sufficient?

Q3 Where did you obtain your CCHF knowledge? a. During my education /b. media/ c. seminars-occupational education/ d. patients whom I followed up.

Q4 Have you ever worked in a clinic where CCHF patients received follow-up care?

Q5 Do you always use gloves during interventions for patients with hemorrhage in the emergency room setting?

Q6 Do you always use protective equipment (masks, gloves, and so on) during interventions for patients who are admitted to the emergency room with nausea, vomiting and enteritis?

Q7 Do you always use protective equipment (masks, gloves, and so on) during interventions for patients who are admitted to the emergency room with active hemorrhage?

Q8 Do you always use gloves during invasive procedures in the emergency room?

Q9 Do you dispose of used sharp equipment and needles in the medical waste box after invasive procedures in the emergency room?

Q10 What is your approach to a suspicious CCHF patient?: a. The patient should be hospitalized immediately/ b. Contact isolation precautions should be implemented/c. Gloves should be used/ d. Facemasks should be used/ e. Sharp equipment and used needles should be disposed of in a medical waste box/ $\mathrm{f}$. Usage of $1 / 10$ diluted bleach is sufficient to disinfect environments that are contaminated with patients' blood and secretions.

Q11 Is CCHF a hemorrhagic viral infection?

Q12 Does CCHF occur due to infection of the human body by the CCHF virus?

Q13 Can the CCHF virus be transmitted to humans by ticks?

Q14 Can all ticks carry and transmit CCHF?

Q15 Can the CCHF virus be transmitted from human to human?

Q16 Is CCHF especially common in the summer?

Q17 Can CCHF be an asymptomatic disease?

Q18 Should adhered ticks on the human body be removed by pouring a substance that kills ticks on the bitten area?

Q19 Should people wear protective clothing and apply insect repellent when in rural and woody areas?

Q20 What are the symptoms and signs of CCHF? (yes/no): Fever, dysuria, headache, hemorrhage, nausea/vomiting, constipation, diarrhea, weakness, diffuse muscle pain, anorexia, cough, rhinorrhea, low white blood cell (WBC) high WBC, anemia, vitamin B12 deficiency, high CK, low platelets, high AST/ALT, positive blood cultures, positive urine cultures.

Q21 What are the transmission methods of CCHF? a. Bite of infected tick/ b. Contact with blood, tissue or secretions of infected animals/ c. Contact with sick people's blood or secretions/ d. Inhalation/ e. Eating the meat of animals that have been bitten by infected ticks.

Q22 Who is at risk for CCHF? a. People living in rural areas and their families/ b. Crop farmers and their families/c. Livestock farmers and their families/d. Soldiers/ e. Campers/f. Scouts/g. Wood workers/ h. People who visit rural and woody areas/ i. Butchers/ j. Abattoir workers/ k. Veterinarians.

Q23 Can CCHF be transmitted nosocomially?

Q24 Should health care workers undergo daily check-ups (of temperature and other symptoms) for 14 days after contact with infected blood and secretions?

Q25 Do you know of an effective and safe vaccine for humans against CCHF?

Q26 Has treating a CCHF patient created risk for you at your job?

Q27 Are you afraid of CCHF, although you are well informed about how it is transmitted?

Q28 Would you prefer not to work with CCFH patients if you could? 
Table 2. General Knowledge Distribution of HCWs About CCHF

\begin{tabular}{|c|c|c|c|c|c|c|c|c|c|c|}
\hline \multirow{2}{*}{ Question } & \multirow{2}{*}{ Answer } & \multicolumn{2}{|c|}{ Doctors } & \multicolumn{2}{|c|}{ Nurses } & \multicolumn{2}{|c|}{ Paramedics } & \multirow{2}{*}{$\mathbf{P}$} & \multicolumn{2}{|c|}{ Total } \\
\hline & & $\mathbf{n}$ & $\%$ & $\mathbf{n}$ & $\%$ & $\mathbf{n}$ & $\%$ & & $\mathbf{n}$ & $\%$ \\
\hline Q1 & Yes & 54 & 100 & 39 & 100 & 16 & 100 & - & 109 & 100 \\
\hline Q2 & Yes & 44 & 81.5 & 28 & 71.8 & 14 & 87.5 & 0.361 & 86 & 78.9 \\
\hline Q3 & Yes & 24 & 44.4 & 22 & 56.4 & 2 & 12.5 & 0.009 & 48 & 44 \\
\hline \multirow[t]{4}{*}{ Q4 } & a & 30 & 55.6 & 14 & 35.9 & 7 & 43.8 & 0.167 & 51 & 46.8 \\
\hline & $\mathrm{b}$ & 0 & 0 & 14 & 35.9 & 0 & 0 & $<0.001$ & 14 & 35.9 \\
\hline & $\mathrm{c}$ & 10 & 18.5 & 19 & 48.7 & 10 & 62.5 & 0.001 & 39 & 35.8 \\
\hline & $\mathrm{d}$ & 10 & 18.5 & 18 & 46.2 & 6 & 37.5 & 0.015 & 34 & 31.2 \\
\hline
\end{tabular}

and occupation (in the ES) of the participants were recorded. The questionnaire consisted of 28 questions about the workers' knowledge levels regarding $\mathrm{CCHF}$ and their approaches to CCHF (Table 1).

The participants' answers were recorded.

\section{Statistical analyses}

STATA 11.0 (College Station, TX, USA) was used for statistical analyses. The data are reported in terms of percentages. Comparisons of answers given by doctors, nurses and paramedics were performed with the program used for statistical evaluation. Data were considered with percentage calculation. Comparison of doctors, nurses and paramadics answers were performed with Chi-square test or Fisher's exact test, as appropriate. Values of $\mathrm{P}<0.05$ were considered statistically significant.

\section{Results}

This study included $37(33.9 \%)$ males and 72 (66.1\%) females, for a total of 109 ES workers. Mean age was $29.6 \pm$ 6.5 (range 19 - 45). Fifty-four (49.54\%) participants were doctors, 39 (35.78\%) were nurses and 16 (14.68\%) were paramedics. All of the participants $(100 \%)$ had heard of CCHF.

Eighty-six (78.9\%) of 109 participants answered that they believed they had adequate knowledge of CCHF. The general knowledge distribution of the $\mathrm{HCWs}$ regarding CCHF is shown in Table 2.

Thirty-three (61.1\%) doctors and 30 (76.9\%) nurses de- clared that they used protective equipment (masks, gloves, and so on) during interventions for patients who were admitted to the ES with active hemorrhage. Thirty $(64.8 \%)$ doctors, nine $(23.1 \%)$ nurses and five $(21.2 \%)$ paramedics were unaware that $1 / 10$ diluted bleach is sufficient for disinfecting environments that are contaminated with the blood and secretions of a patient with suspected CCHF. Seventeen (40.7\%) doctors, 22 (56.4\%) nurses and 11 (69.7\%) paramedics did not know that CCHF may be asymptomatic. Seventeen (15.6\%) participants did not know that adhered ticks on the human body should not be removed by pouring a substance that kills ticks on the bitten area. The knowledge level distributions of these HCWs in terms of transmission prevention and the approach to CCHF patients are shown in Table 3. Seventy (64.2\%) HCWs said that positive blood cultures are a laboratory finding in CCHF. Details regarding the HCWs' answers regarding the symptoms and laboratory findings of CCHF are provided in Table 4. Fifty (45.9\%) of the participants said that CCHF can be transmitted by inhalation. The HCWs' knowledge level distribution regarding methods of transmission and populations at risk for CCHF is detailed in Table 5.

Ten $(9.2 \%)$ participants did not think that CCHF was associated with a transmission risk for hospital-borne infections. One hundred (91.7\%) HCWs believed that caring for a CCHF patient created risk at their job, and $62(56.9 \%)$ declared that they would prefer not to work with CCHF patients if that were an option. The personnel approach distribution of HCWs (as hospital workers) for CCHF is displayed in Table 6.

Among $86(78.9 \%)$ HCWs who believed that their knowledge about CCHF was sufficient, 50 (58.1\%) declared 
Table 3. Distribution of Participants Who Answered "Yes" to Questions About Prevention of Transmission and Approach to Patients

\begin{tabular}{|c|c|c|c|c|c|c|c|c|c|}
\hline \multirow{2}{*}{ Question } & \multicolumn{2}{|c|}{ Doctors } & \multicolumn{2}{|c|}{ Nurses } & \multicolumn{2}{|c|}{ Paramedics } & \multirow{2}{*}{$\mathbf{P}$} & \multicolumn{2}{|c|}{ Total } \\
\hline & $\mathbf{n}$ & $\%$ & $\mathbf{n}$ & $\%$ & $\mathbf{n}$ & $\%$ & & $\mathbf{n}$ & $\%$ \\
\hline Q5 & 49 & 90.7 & 39 & 100 & 16 & 100 & 0.069 & 104 & 95.4 \\
\hline Q6 & 33 & 61.1 & 35 & 89.7 & 16 & 100 & $<0.001$ & 84 & 77.1 \\
\hline Q7 & 33 & 61.1 & 30 & 76.9 & 16 & 100 & 0.003 & 79 & 72.5 \\
\hline Q8 & 49 & 90.7 & 39 & 100 & 16 & 100 & 0.069 & 104 & 95.4 \\
\hline Q9 & 47 & 87 & 29 & 74.4 & 11 & 68.8 & 0.158 & 87 & 79.8 \\
\hline Q10a & 35 & 64.8 & 37 & 94.9 & 11 & 68.8 & 0.001 & 83 & 76.2 \\
\hline Q10b & 52 & 96.3 & 39 & 100 & 16 & 100 & 0.642 & 107 & 98.2 \\
\hline Q10c & 54 & 100 & 39 & 100 & 16 & 100 & - & 109 & 100 \\
\hline Q10d & 54 & 100 & 39 & 100 & 16 & 100 & - & 109 & 100 \\
\hline Q10e & 34 & 63 & 33 & 84.6 & 9 & 56.3 & 0.036 & 76 & 69.7 \\
\hline Q10f & 19 & 35.2 & 30 & 76.9 & 11 & 68.8 & $<0.001$ & 60 & 55.1 \\
\hline Q11 & 54 & 100 & 37 & 94.9 & 16 & 100 & 0.252 & 107 & 98.2 \\
\hline Q12 & 50 & 92.6 & 39 & 100 & 16 & 100 & 0.200 & 105 & 96.3 \\
\hline Q13 & 54 & 100 & 37 & 94.9 & 16 & 100 & 0.252 & 107 & 98.2 \\
\hline Q14 & 11 & 20.4 & 11 & 28.2 & 5 & 31.3 & 0.558 & 27 & 24.8 \\
\hline Q15 & 54 & 100 & 37 & 94.9 & 16 & 100 & 0.252 & 107 & 98.2 \\
\hline Q16 & 54 & 100 & 37 & 94.9 & 16 & 100 & 0.252 & 107 & 98.2 \\
\hline Q17 & 32 & 59.3 & 17 & 43.6 & 5 & 31.3 & 0.094 & 54 & 49.5 \\
\hline Q18 & 5 & 9.3 & 7 & 18 & 5 & 31.3 & 0.091 & 17 & 15.6 \\
\hline Q19 & 54 & 100 & 39 & 100 & 16 & 100 & - & 109 & 100 \\
\hline
\end{tabular}

that they would prefer not to follow patients with $\mathrm{CCHF}(\mathrm{P}$ $=0.608)$.

\section{Discussion}

HCWs are an important risk group for CCHF infection in endemic areas. Infected patients should be isolated, and barrier nursing techniques should be used. Strict universal precautions are necessary, and health care workers should wear protective clothing such as disposable gowns, gloves and masks, as well as goggles or face shields. During procedures that may produce aerosols, an N95 mask should be worn. Human infections are mainly caused by direct contact with blood or tissues of viremic hosts, as well as by tick bites or crushing infected ticks with unprotected hands. In endemic areas, high-risk groups include persons who have occupational contact with livestock and other animals, such 
Table 4. Distribution of Participants Who Answered "Yes" to Questions About Symptoms and Laboratory Findings of CCHF (Q20)

\begin{tabular}{|c|c|c|c|c|c|c|c|c|c|}
\hline \multirow{2}{*}{ Question } & \multicolumn{2}{|c|}{ Doctors } & \multicolumn{2}{|c|}{ Nurses } & \multicolumn{2}{|c|}{ Paramedics } & \multirow{2}{*}{$\mathbf{P}$} & \multicolumn{2}{|c|}{ Total } \\
\hline & $\mathbf{n}$ & $\%$ & $\mathbf{n}$ & $\%$ & $\mathbf{n}$ & $\%$ & & $\mathbf{n}$ & $\%$ \\
\hline High fever & 54 & 100 & 39 & 100 & 16 & 100 & - & 109 & 100 \\
\hline Dysuria & 10 & 18.5 & 10 & 25.6 & 2 & 12.5 & 0.516 & 22 & 20.2 \\
\hline Headache & 52 & 96.3 & 30 & 76.9 & 9 & 56.3 & $<0.001$ & 91 & 83.5 \\
\hline Hemorrhage & 54 & 100 & 33 & 84.6 & 16 & 100 & 0.004 & 103 & 94.5 \\
\hline Nausea/vomiting & 51 & 94.4 & 37 & 94.9 & 16 & 100 & 1.000 & 104 & 95.4 \\
\hline Constipation & 0 & 0 & 8 & 20.5 & 0 & 0 & $<0.001$ & 8 & 7.3 \\
\hline Diarrhea & 37 & 68.5 & 32 & 82.1 & 16 & 100 & 0.013 & 85 & 78 \\
\hline Weakness & 54 & 100 & 39 & 100 & 16 & 100 & - & 109 & 100 \\
\hline Diffuse muscle pain & 51 & 94.4 & 33 & 84.6 & 16 & 100 & 0.136 & 100 & 91.7 \\
\hline Anerexia & 48 & 88.9 & 37 & 94.8 & 11 & 68.8 & 0.034 & 96 & 88.1 \\
\hline Cough & 22 & 40.7 & 7 & 18 & 5 & 31.3 & 0.065 & 34 & 31.2 \\
\hline Rinorrhea & 24 & 44.4 & 16 & 41 & 12 & 75 & 0.058 & 52 & 47.7 \\
\hline Low WBC & 32 & 59.3 & 27 & 69.2 & 2 & 12.5 & $<0.001$ & 61 & 56 \\
\hline High WBC & 27 & 50 & 19 & 48.7 & 9 & 56.3 & 0.875 & 55 & 50.5 \\
\hline Vit B12 deficiency & 2 & 3.7 & 17 & 43.6 & 5 & 31.3 & $<0.001$ & 24 & 22 \\
\hline High CK level & 40 & 74.1 & 27 & 69.2 & 11 & 68.8 & 0.846 & 78 & 71.6 \\
\hline High LDH & 39 & 72.2 & 27 & 69.2 & 9 & 56.3 & 0.479 & 75 & 68.8 \\
\hline Low thrombocytes & 51 & 94.4 & 25 & 64.1 & 6 & 37.5 & $<0.001$ & 82 & 75.2 \\
\hline High AST/ALT & 54 & 100 & 36 & 92.3 & 14 & 87.5 & 0.026 & 104 & 95.4 \\
\hline Positive blood cultures & 34 & 63 & 25 & 64.1 & 11 & 68.8 & 0.914 & 70 & 64.2 \\
\hline Positive urine cultures & 18 & 33.3 & 21 & 53.9 & 7 & 43.8 & 0.141 & 46 & 42.2 \\
\hline
\end{tabular}

as farmers, livestock owners, abattoir workers and veterinarians. Recreational activities such as hiking and camping in endemic areas are also risk factors for tick bites. As the $\mathrm{CCHF}$ virus is destroyed by tissue acidification and does not survive cooking, meat consumption is safe. The ratio of subclinical to clinical CCHF cases is approximately 5:1, and $80 \%$ of infections are asymptomatic. The nosocomial route is an important transmission mechanism for CCHF. HCWs caring for patients with $\mathrm{CCHF}$ are a major risk group. Direct transmission is thought to occur through contact of broken skin with viremic blood or other body fluids. Interventions for gastrointestinal bleeding, surgery on patients with occult disease, needle stick injuries and unprotected handling of infected materials are high-risk activities. Case fatality rates among nosocomial cases tend to be higher than in community-acquired cases, which may be related to the viral inoculums [15].

Emergency room HCWs constitute a high-risk group for blood- and secretion-borne infections [9]. For many emergency room patients, it is often difficult to obtain a detailed medical history because of time constraints. For example, when a patient is admitted to the emergency room with hem- 
Table 5. Knowledge Level Distribution of HCWs Regarding Transmission Methods and At-Risk Populations for $\mathrm{CCHF}$

\begin{tabular}{|c|c|c|c|c|c|c|c|c|c|c|}
\hline \multirow{2}{*}{ Question } & \multirow{2}{*}{ Answer } & \multicolumn{2}{|c|}{ Doctors } & \multicolumn{2}{|c|}{ Nurses } & \multicolumn{2}{|c|}{ Paramedics } & \multirow{2}{*}{$\mathbf{P}$} & \multicolumn{2}{|c|}{ Total } \\
\hline & & $\mathbf{n}$ & $\%$ & $\mathbf{n}$ & $\%$ & $\mathbf{n}$ & $\%$ & & $\mathbf{n}$ & $\%$ \\
\hline \multirow[t]{5}{*}{ Q21 } & $\mathrm{a}$ & 54 & 100 & 39 & 100 & 16 & 100 & - & 109 & 100 \\
\hline & $\mathrm{b}$ & 46 & 85.2 & 30 & 76.9 & 14 & 87.5 & 0.577 & 90 & 82.6 \\
\hline & $\mathrm{c}$ & 49 & 90.7 & 37 & 94.9 & 16 & 0 & 0.655 & 102 & 93.6 \\
\hline & $\mathrm{d}$ & 25 & 46.3 & 11 & 28.2 & 14 & 87.5 & $<0.001$ & 50 & 45.9 \\
\hline & e & 7 & 13 & 9 & 23.1 & 7 & 43.8 & 0.028 & 23 & 21.1 \\
\hline \multirow[t]{11}{*}{ Q22 } & $\mathrm{a}$ & 54 & 100 & 39 & 100 & 16 & 100 & - & 109 & 100 \\
\hline & $\mathrm{b}$ & 47 & 87 & 39 & 100 & 16 & 100 & 0.033 & 102 & 93.6 \\
\hline & $\mathrm{c}$ & 54 & 100 & 39 & 100 & 16 & 100 & - & 109 & 100 \\
\hline & $\mathrm{d}$ & 28 & 51.9 & 21 & 53.9 & 11 & 68.8 & 0.482 & 60 & 55.1 \\
\hline & $\mathrm{e}$ & 54 & 100 & 37 & 94.9 & 16 & 100 & 0.252 & 107 & 98.2 \\
\hline & $\mathrm{f}$ & 49 & 90.7 & 34 & 87.2 & 16 & 100 & 0.438 & 99 & 90.8 \\
\hline & $\mathrm{g}$ & 52 & 96.3 & 37 & 94.9 & 16 & 100 & 1.000 & 105 & 96.3 \\
\hline & $\mathrm{h}$ & 51 & 94.4 & 36 & 92.3 & 16 & 100 & 0.739 & 103 & 94.5 \\
\hline & $\mathrm{i}$ & 34 & 63 & 25 & 64 & 7 & 43.8 & 0.354 & 66 & 60.6 \\
\hline & $\mathrm{j}$ & 47 & 87 & 29 & 74.4 & 11 & 68.8 & 0.158 & 87 & 79.8 \\
\hline & $\mathrm{k}$ & 50 & 92.6 & 34 & 87.2 & 16 & 100 & 0.347 & 100 & 91.7 \\
\hline
\end{tabular}

orrhage, contact with the patient begins before laboratory evaluations can be obtained. Therefore, emergency room physicians, nurses and other HCWs must begin care before having definitive information about a patient's previous health history and current diagnosis. Despite these complicating factors, HCWs are responsible for protecting themselves against infectious diseases. Therefore, knowledge of infectious diseases and their transmission methods, especially in endemic regions, is important for HCWs. A young emergency resident physician died in October 2012 due to a needle stick injury while caring for a CCHF patient in Turkey [16]. There are a limited number of studies regarding CCHF knowledge levels among HCWs in the literature. In this study, we aimed to investigate CCHF knowledge levels among emergency department HCWs in an endemic region.

In Rahnavardi et al's cross-sectional study, 209 HCWs from three hospitals in a region where CCHF was common were included. In this study, 11 (5.8\%) participants had heard of CCHF. In our study, all of the participants (100\%) had heard about CCHF. These findings suggested that being a physician and relying on academic material rather than local media were independently and significantly associated with higher knowledge levels. Education levels and laboratory staff attitudes were also significant factors. Forty-four percent of the study group wore gloves and masks for contact with CCHF patients, and $22 \%$ failed to observe any safety measures [17]. In our study, $86(78.9 \%)$ of 109 participants believed that their knowledge levels regarding $\mathrm{CCHF}$ were sufficient.

Fifty-four (49.54\%) participants were doctors, 39 $(35.78 \%)$ were nurses and $16(14.68 \%)$ were paramedics. Thirty-three (61.1\%) doctors and $30(76.9 \%)$ nurses declared that they used protective equipment (masks, gloves, and so on) during interventions for patients who were admitted to the emergency department with active hemorrhage. Thirty $(64.8 \%)$ doctors, nine $(23.1 \%)$ nurses and five $(21.2 \%)$ para- 
Table 6. Distribution of Participants Who Answered "Yes" to Questions About the Approach to CCHF as a Hospital Worker

\begin{tabular}{|c|c|c|c|c|c|c|c|c|c|}
\hline \multirow{2}{*}{ Question } & \multicolumn{2}{|c|}{ Doctors } & \multicolumn{2}{|c|}{ Nurses } & \multicolumn{2}{|c|}{ Paramedics } & \multirow{2}{*}{$\mathbf{P}$} & \multicolumn{2}{|c|}{ Total } \\
\hline & $\mathbf{n}$ & $\%$ & $\mathbf{n}$ & $\%$ & $\mathbf{n}$ & $\%$ & & $\mathbf{n}$ & $\%$ \\
\hline Q23 & 51 & 94.4 & 32 & 82 & 16 & 100 & 0.057 & 99 & 90.8 \\
\hline Q24 & 51 & 94.4 & 36 & 92.3 & 16 & 100 & 0.739 & 103 & 94.5 \\
\hline Q25 & 52 & 96.3 & 39 & 100 & 16 & 100 & 0.642 & 107 & 98.2 \\
\hline Q26 & 47 & 87 & 37 & 94.9 & 16 & 100 & 0.305 & 100 & 91.7 \\
\hline Q27 & 46 & 85.2 & 32 & 85 & 16 & 100 & 0.204 & 94 & 86.2 \\
\hline Q28 & 36 & 66.7 & 15 & 38.5 & 11 & 68.8 & 0.015 & 62 & 56.9 \\
\hline
\end{tabular}

medics were unaware that $1 / 10$ diluted bleach is adequate for disinfecting environments that are contaminated with the blood and secretions of a suspected CCHF patient. Seventeen (40.7\%) doctors, 22 (56.4\%) nurses and 11 (69.7\%) paramedics were unaware that CCHF may be asymptomatic. Seventy (64.2\%) HCWs said that positive blood cultures are a laboratory finding in CCHF. Fifty $(45.9 \%)$ of the participants said that CCHF can be transmitted by inhalation. Paramedics were more compliant than doctors and nurses with preventative measures.

Ten (9.2\%) participants did not think that CCHF could be transmitted nosocomially. One hundred $(91.7 \%) \mathrm{HCWs}$ believed that caring for a CCHF patient created workplace risk, and $62(56.9 \%)$ declared that they would prefer not to work with CCFH patients if possible.

Yilmaz et al attempted to determine knowledge levels, attitudes and practices regarding CCHF in people visiting a tertiary care hospital in an endemic city in Turkey. They provided questionnaires to the relatives or guardians of patients who were admitted to pediatric outpatient clinics and studied 1,034 participants. According to these authors, the media are the most useful source of information on this disease. They also described insufficient knowledge regarding CCHF in the normal population and suggested that the health, agriculture and media sectors can improve public knowledge and awareness of CCHF [18].

\section{Conclusions}

In the 10th year after the first CCHF outbreaks in Turkey, we demonstrate that ES HCWs in endemic regions have insuffi- cient knowledge about this disease. We believe that seminars and education about CCHF and its transmission methods may be helpful for ES HCWs; furthermore, undergraduate curricula for all health-related courses should be reviewed to ensure effective education on this topic. Most CCHF patients first present in the emergency room. Therefore, techniques that protect against transmission of this disease, including gloves (especially baricidal gloves), face masks, face visors and box coats, should be explained to ES HCWs, and the use of these techniques should be encouraged.

This report describes the first study of CCHF knowledge levels among emergency room HCWs in an endemic region. In the future, comprehensive studies may be helpful to prevent the deaths of HCWs due to this disease.

\section{Acknowledgment}

This study was performed with the local ethics committee approval.

\section{Conflict of Interest}

All authors declare that they have no conflict of interest.

\section{Author Contributions}

SY, CK and AE carried out the conception and design of the study. SO and AEK acquised, analyzed and interpreted 
the data. AE carried out the statistical analyse. SY and AE drafted the article and revised it critically for important intellectual content. All authors read and approved the final manuscript.

\section{References}

1. Simpson DI. Viral haemorrhagic fevers of man. Bull World Health Organ. 1978;56(6):819-832.

2. Williams RJ, Al-Busaidy S, Mehta FR, Maupin GO, Wagoner KD, Al-Awaidy S, Suleiman AJ, et al. Crimean-congo haemorrhagic fever: a seroepidemiological and tick survey in the Sultanate of Oman. Trop Med Int Health. 2000;5(2):99-106.

3. Bakir M, Ugurlu M, Dokuzoguz B, Bodur H, Tasyaran MA, Vahaboglu H, Turkish CSG. Crimean-Congo haemorrhagic fever outbreak in Middle Anatolia: a multicentre study of clinical features and outcome measures. J Med Microbiol. 2005;54(Pt 4):385-389.

4. Gozalan A, Esen B, Fitzner J, Tapar FS, Ozkan AP, Georges-Courbot MC, Uzun R, et al. Crimean-Congo haemorrhagic fever cases in Turkey. Scand J Infect Dis. 2007;39(4):332-336.

5. Flick R, Whitehouse CA. Crimean-Congo hemorrhagic fever virus. Curr Mol Med. 2005;5(8):753-760.

6. Vorou R, Pierroutsakos IN, Maltezou HC. Crimean-Congo hemorrhagic fever. Curr Opin Infect Dis. 2007;20(5):495-500.

7. Ergonul O, Zeller H, Celikbas A, Dokuzoguz B. The lack of Crimean-Congo hemorrhagic fever virus antibodies in healthcare workers in an endemic region. Int $\mathrm{J}$ Infect Dis. 2007;11(1):48-51.

8. Beltrami EM, Williams IT, Shapiro CN, Chamberland ME. Risk and management of blood-borne infections in health care workers. Clin Microbiol Rev. 2000;13(3):385-407.

9. Werman HA, Gwinn R. Seroprevalence of hepatitis B and hepatitis $\mathrm{C}$ among rural emergency medical care personnel. Am J Emerg Med. 1997;15(3):248-251.
10. Mardani M. Nosocomial CrimeaneCongo haemorrhagic fever in Iran (1999-2000). In: 11th European congress of clinical microbiology and infectious disease, Istanbul, Turkey. 2001:P1044.

11. Burney MI, Ghafoor A, Saleen M, Webb PA, Casals J. Nosocomial outbreak of viral hemorrhagic fever caused by Crimean Hemorrhagic fever-Congo virus in Pakistan, January 1976. Am J Trop Med Hyg. 1980;29(5):941947.

12. van Eeden PJ, Joubert JR, van de Wal BW, King JB, de Kock A, Groenewald JH. A nosocomial outbreak of Crimean-Congo haemorrhagic fever at Tygerberg Hospital. Part I. Clinical features. S Afr Med J. 1985;68(10):711-717.

13. Fisher-Hoch SP, McCormick JB, Swanepoel R, Van Middlekoop A, Harvey S, Kustner HG. Risk of human infections with Crimean-Congo hemorrhagic fever virus in a South African rural community. Am J Trop Med Hyg. 1992;47(3):337-345.

14. Altaf A, Luby S, Ahmed AJ, Zaidi N, Khan AJ, Mirza S, McCormick J, et al. Outbreak of Crimean-Congo haemorrhagic fever in Quetta, Pakistan: contact tracing and risk assessment. Trop Med Int Health. 1998;3(11):878882.

15. Erbay A. Crimean-Congo Hemorrhagic Fever Virus In: Liu D eds. Molecular detection of human viral pathogens. Boca Raton, FL, US: CRC Press Taylor and Francis Group, 2010:617-629.

16. http://www.medimagazin.com.tr/medimagazin/trgenc-asistan-kurtarilamadi-676-606-11889.html. Date:04.11.2013.

17. Rahnavardi M, Rajaeinejad M, Pourmalek F, Mardani M, Holakouie-Naieni K, Dowlatshahi S. Knowledge and attitude toward Crimean-Congo haemorrhagic fever in occupationally at-risk Iranian healthcare workers. J Hosp Infect. 2008;69(1):77-85.

18. Yilmaz R, Ozcetin M, Erkorkmaz U, Ozer S, Ekici F. Public Knowledge and Attitude toward Crimean Congo Hemorrhagic Fever in Tokat Turkey. Iran J Arthropod Borne Dis. 2009;3(2):12-17. 